\title{
Verification of Infection Prevention Control Using a Spatial Random Walk Model
}

\author{
Toshiaki Ichinose ${ }^{1,2}$, Danhe Tian ${ }^{3} \&$ Yifeng $\mathrm{Li}^{4}$ \\ ${ }^{1}$ Center for Social and Environmental Systems Research, National Institute for Environmental Studies, Tsukuba City, \\ Japan \\ ${ }^{2}$ Graduate School of Environmental Studies, Nagoya University, Nagoya City, Japan \\ ${ }^{3}$ Graduate School of Science and Technology, University of Tsukuba / Center for Social and Environmental Systems \\ Research, National Institute for Environmental Studies, Tsukuba City, Ibaraki, Japan \\ ${ }^{4}$ (Former Affiliation) Graduate School of Life and Environmental Sciences, University of Tsukuba / (Former Affiliation) \\ Center for Social and Environmental Systems Research, National Institute for Environmental Studies, Tsukuba City, \\ Ibaraki, Japan \\ Correspondence: Toshiaki Ichinose, Center for Social and Environmental Systems Research, National Institute for \\ Environmental Studies, 16-2 Onogawa, Tsukuba City, Ibaraki, 305-8506, Japan.
}

Received: August 4, 2020

doi:10.11114/ijsss.v8i6.4955

\author{
Accepted: August 28, 2020 \\ Available online: September 30, 2020 \\ URL: https://doi.org/10.11114/ijsss.v8i6.4955
}

\begin{abstract}
To stop pandemic of the 2019 novel coronavirus (COVID-19), "an 80 percent reduction of person to person contact opportunities" was proposed by the Japanese government. This guideline was based on the result of macroscopic differential equation model akin to the SIR (Susceptible-Infected-Recovered) model. For the purpose of indicating person to person's infection mechanism intuitively, we built a new model to calculate infections between two persons who are in contact each other. This model adopted a spatial random walk model to express random movement of people in a specific 2-D geographical space. This model was applied to verify the effect of the proposed infection control procedure, "80 percent reduction". The result of the numerical simulation supported a proposed infection control procedure of "an 80 percent reduction" derived by the SIR model.
\end{abstract}

Keywords: COVID-19, epidemics, infection control, random walk model, spatial model

\section{Introduction}

SARS-CoV-2 is a SARSr-CoV coronavirus strain that causes the disease COVID-19 (Gorbalenya et al., 2020). It was first identified in Wuhan area of China in December 2019 (Zhou et al., 2020) and by xx date has since caused a global pandemic of COVID-19. In Japan, the number of infections started to increase from mid-March of 2020, among people returning from overseas and among persons in whom the route of infection was unknown, leading an outbreak of a full-scale epidemic.

On the 7th of April of 2020, a state of emergency was declared in seven prefectures of Japan including the Tokyo metropolitan area (https://corona.go.jp/news/pdf/kinkyujitai_sengen_0407.pdf; in the Home Page of the Japanese Cabinet Secretariat, referenced on 27 April of 2020). On the evening of 16th April of 2020, this was extended to the entire country. To prevent the spread of infection, person-to-person contact by ought to be reduced by $80 \%$ based on calculations done by Prof. Nishiura (Hokkaido University) and others in the Cluster Response Team of the Japanese Ministry of Health, Labour and Welfare (MHLW) using numerical model simulations. Based on news reports (e.g. https://www.mhlw.go.jp/content/10900000/000624048.pdf; in the Home Page of the MHLW, referenced on 22 April of 2020), the numerical model was based on the SIR model (Kermack and McKendrick 1927), a classic set of equations that provide a deterministic description of the short-term spread of infection in an epidemic. The SIR model assumes there are three statuses of populations in an epidemic; Susceptible (S), Infected (I), and Recovered (R). Susceptible individuals progress to Infected status as a constant proportion of the product of the number of Susceptible and Infected individuals, after which Infected individuals progress to Recovered status at a constant rate, with the model's development over time expressed as a continuous dynamical system that is described by an ordinary differential equation. However, the actual 
spread of infection occurs in two-dimensional (2-D) spaces in specific regions, and therefore results calculated solely from macroscopic differential equations are not intuitively easy to understand.

Modelling studies on the spread of infection include analyses simulating the movement of people and pathogens in such 2-D spaces akin to the horizontal flow and dispersion of physical substances (Smallman-Raynor, Cliff \& Barford, 2015; Haggett, 1976; Reluga, Medlock \& Galvani, 2006; Birrell et al., 2016; Simoes, 2005; Chen et al., 2005; Fitzgibbon et al., 2019). For example, Simoes (2005) used an agent-based model (ABM) focused on infected individuals and Fitzgibbon et al. (2019) used a geographical information system (GIS)-based model. However, a limited number of such studies, clearly describe the system of time evolution equations underlying their model.

In this study, we used a random-walk model (Nicosia et al., 2017; Escaff et al., 2018) to express the movements of random individuals in the 2-D space (geographic space) within a specific area. We then developed a model to calculate the probability of infection between two individuals who come into contact with each other, with a view to simplify this mechanism. We then used this model to investigate the efficacy of a $80 \%$ reduction in person-to-person contact in reducing infection transmission.

\section{Structures of the Model}

To analyze this individual process of infection transmission between people in a microcosm, we set up fields illustrating 100 islanders on an isolated island as shown in Figure 1. We assumed 100 people were located a square island isolated outside this world and allocated each person to each cell of a $10 \times 10$ grid. We aimed to simulate people's movements by using a random-walk model of the spread of infection as all the 100 inhabitants of this island (including those initially infected) randomly moved about the island. Each inhabitant (numbered from 1 to 100) moved between cells at a probability based on regulations shown in Figure 2. The probability of moving to each of the nine possible cells, including the initially allocated cell, ranged from $9.00 \%$ and $12.25 \%$. If the probability of moving to each of the nine possible cells was equal, this probability would be $11.11 \%$ in each case. However, in this simulation the direction in which each individual moved in the east-west and north-south orientations was determined based on a random number between 0 and 1 , where moving backward would make this probability $<0.35$, forward $>0.65$, and staying in the same place would make this probability $0.35-0.65$. The results of this simulation are shown in the probability distribution in Figure 2. Nevertheless, if this pattern resulted in a move to a cell outside the grid from a cell at the margin, the east-west (right-left) or north-south (up-down) direction was forcibly reversed (akin to bouncing off a wall).

\begin{tabular}{|c|c|c|c|c|c|c|c|c|c|}
\hline 1 & 2 & 3 & 4 & 5 & 6 & 7 & 8 & 9 & 10 \\
\hline 11 & 12 & 13 & 14 & 15 & 16 & 17 & 18 & 19 & 20 \\
\hline 21 & 22 & 23 & 24 & 25 & 26 & 27 & 28 & 29 & 30 \\
\hline 31 & 32 & 33 & 34 & 35 & 36 & 37 & 38 & 39 & 40 \\
\hline 41 & 42 & 43 & 44 & 45 & 46 & 47 & 48 & 49 & 50 \\
\hline 51 & 52 & 53 & 54 & 55 & 56 & 57 & 58 & 59 & 60 \\
\hline 61 & 62 & 63 & 64 & 65 & 66 & 67 & 68 & 69 & 70 \\
\hline 71 & 72 & 73 & 74 & 75 & 76 & 77 & 78 & 79 & 80 \\
\hline 81 & 82 & 83 & 84 & 85 & 86 & 87 & 88 & 89 & 90 \\
\hline 91 & 92 & 93 & 94 & 94 & 95 & 97 & 98 & 99 & 100 \\
\hline
\end{tabular}

Figure 1. 100 islanders on an isolated island. 5 islanders are infected at the initial stage

Note: $\mathrm{t}=0$. 




Figure 2. Regulations of movement in the random walking

Note: Left: Probabilities of presence at the next time-step. Right: Reflections at the margins. NRN $=<0.35$ (Backward), $0.35<\mathrm{NRN}<0.65$ (Stay), NRN $>=0.65$ (Forward), On X (W-E) and $\mathrm{Y}(\mathrm{S}-\mathrm{N})$ each.

The initially infected inhabitants were designated as the islander in cell 55, who was located roughly in the center of the grid in Figure 1, and those in four other cells $(45,54,56,65)$ to the north, west, east, and south (up, left, right, down) of islander number 55, respectively. Following this, any inhabitant who occupied the same cell as any of the five originally infected islanders had a fixed probability of coming into contact with the infected islander and therefore becoming infected. For example, the track followed by islander 55 during the ten subsequent time-steps is shown in Figure 3.

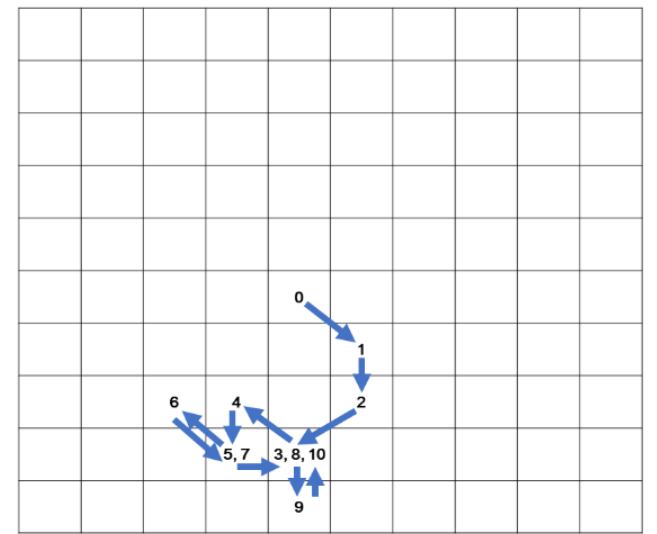

Figure 3. Random walking track of 10 time-steps

Note: The islander of No. 55

Because in real life infected individuals do not move around when they develop symptoms or receive a positive diagnosis, this is believed to limit subsequent infection transmission. Therefore, we also incorporated cessation of movement and removal from the system of persons who are infected in this. Simulations that cover long periods should also take into account the process of recovery and becoming virus-free. However, this simulation that was modelling the worst-case scenario did not incorporate these elements. Therefore, our model did not express the Recovered status from the SIR model but only focused on the initial spread of infection.

When the population density in the grid cells was sufficiently low that even if two inhabitants occupied the same grid cell they did not come into direct contact, infection would not result. Whether or not inhabitants actually came into contact within the cells of the grid was handled as a sub-grid scale phenomenon, and a contact (infection) -rate parameter was be set. In this study, we re-envisaged this parameter as the percentage decrease in contact between inhabitants.

\section{Results}

As previously described, in this study we assumed that contact between inhabitants resulted in infection. 
Figure 4 compares the spread of infection at time-step 10 under several different scenarios. In a scenario where there was free movement after a diagnosis of infection and no decrease in contact rates, 93 out of 100 inhabitants became infected. Some cells (yellow and red) contained four or more infected individuals.

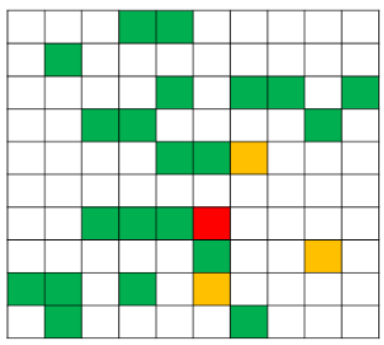

No limitations of movement. No limitations of $\mathrm{P}$ to $\mathrm{P}$ contact.

93 infected among 100

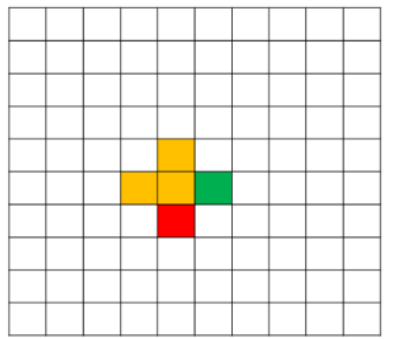

No movement of infected. No limitations of $\mathrm{P}$ to $\mathrm{P}$ contact.

22 infected among 100

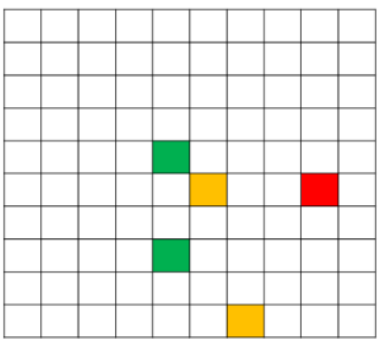

No limitations of movement. 80 percent reduction of $\mathrm{P}$ to $\mathrm{P}$ contact.

32 infected among 100

Figure 4. Comparison of infection between cases (at $\mathrm{t}=10$ )

Note: Numbers of infected: More than 6 as red, 4 to 5 as yellow, 2 to 3 as green.

In a scenario where no movement was permitted after diagnosis of infection, and the rate of person to person contact was not decreased, although some cells contained four or more infected individuals, only 22 out of 100 inhabitants were infected.

In a scenario where free movement was permitted after diagnosis of infection, but the rate of person-to-person contact was decreased by $80 \%$, only 32 out of 100 inhabitants were infected.

As the basic behavior of our model closely aligned to our assumptions, we expanded it to a population of 10,000 people in a grid of 100 x 100 cells. Similar to Figure 1, the initial infected inhabitants were designated as the islander in cell 5050, roughly in the center of the island, and in the four cells $(4950,5049,5051,5150)$ to its' to the north, west, east, and south (up, left, right, down), respectively. The number of time-steps was extended to 100 (Figure 5).

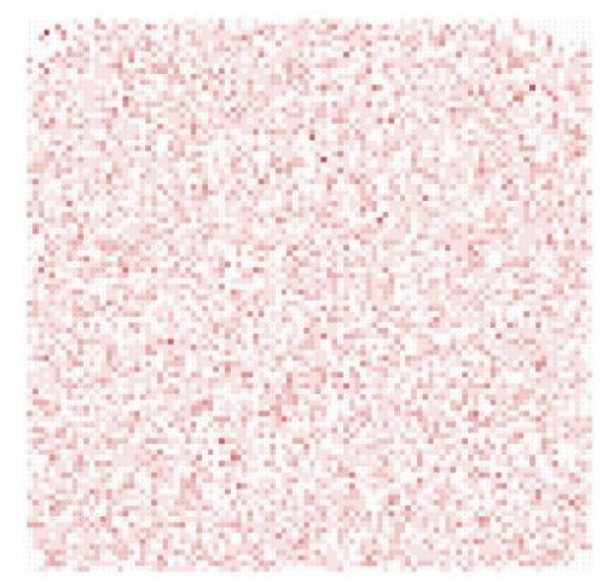

Figure 5. A case of 10000 islanders (100 time-steps) (at $\mathrm{t}=100)$

Note: The maximum number of infected in a grid cell: 9.

Figure 6 compares the spread of infection under several different scenarios. In the absence of any restrictions on post-infection movement or person-to-person contact, the infection spread exponentially, but by the end of the simulation it showed signs of levelling off, possibly because of the small number of uninfected individuals. 


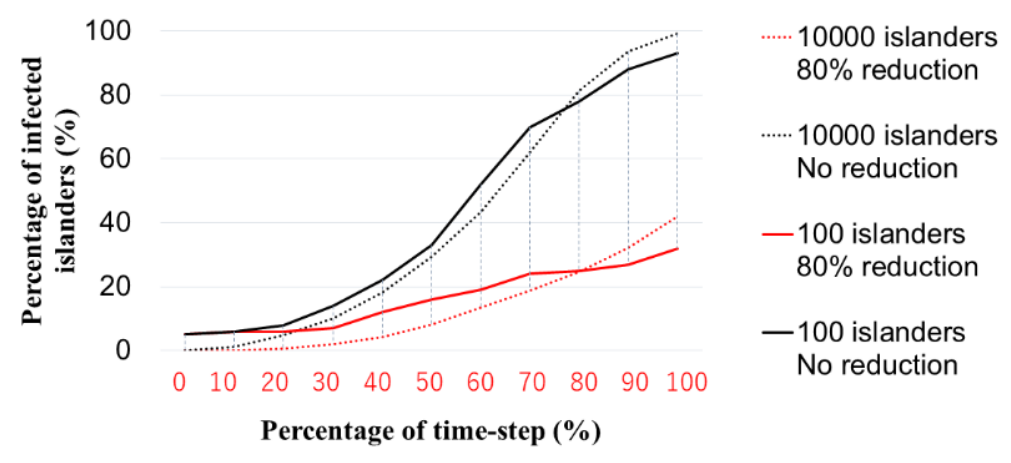

Figure 6. Spread of infection in each scenario: 100 islanders (10 time-steps) vs 10000 islanders (100 time-steps)

Note: Including the initial 5 infected ( $\mathrm{t}=0$ to 10$)$. Less than $1.25 \%$ in the case of No-No (10000 islanders, 100 time-steps).

When the contact rate was reduced by $80 \%$ without any restrictions on post-infection movement, this was effective in limiting the spread of infection with only $40 \%$ of islanders infected at the end of the simulation. When restrictions on posts-infection movement were imposed without reducing the contact rate, the proportion of infected inhabitants in the 10,000-person simulation was kept below 1.25\%. It can't be explicitly expressed in the vertical scale of Figure 6 and 7.

These results indicate that although both isolating infected individuals (restriction of movement) and reducing contact are important, restricting movement has by far the greatest effect.

Figure 7 shows the comparative infection rates at the final time-step for different rates of contact reduction. With unrestricted post-infection movement, the spread of infection was almost contained when contact was reduced by $90 \%$.

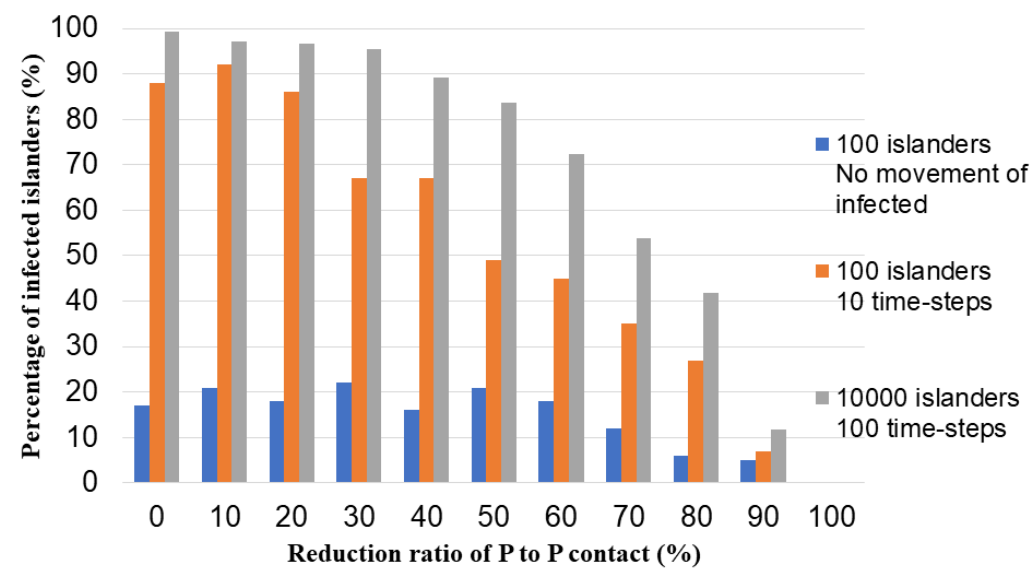

Figure 7. Infected rates at the final time-step in each scenario: 100 islanders (10 time-steps) vs 10000 islanders (100 time-steps)

Note: Not including the initial 5 infected. Less than $1.25 \%$ in the case of No-No (10000 islanders, 100 time-steps).

It is possible that reducing the rate of person-to-person contact appears to have little effect in the early stages of infection (simulation steps) because the diversity of movement generated by random numbers means that the focus is on whether or not individuals occupy the same cell, rather than whether or not they are in contact within the same cell.

\section{Discussion}

In this study we postulate that the figures intermediate between the two cases of post-infection movement or no movement should perhaps be considered as a more realistic scenario. For this reason, we did not express post-infection isolation in terms of the cessation of movement or removal from the system.

The $80 \%$ reduction ought to be applied to individuals who are either uninfected or unaware that they are infected, and 
not people who are aware that they are infected.

The results of our numerical simulation of a temporospatial random walk model in this study are consistent with the rationale for the $80 \%$ reduction derived from a different approach, namely, the SIR model.

Author Contributions: Conceptualization, Toshiaki Ichinose; Data curation, Toshiaki Ichinose; Formal analysis, Toshiaki Ichinose; Investigation, Toshiaki Ichinose, Danhe Tian and Yifen Li; Methodology, Toshiaki Ichinose; Project administration, Toshiaki Ichinose; Resources, Toshiaki Ichinose; Software, Toshiaki Ichinose; Validation, Toshiaki Ichinose; Visualization, Danhe Tian; Writing - original draft, Toshiaki Ichinose; Writing - review \& editing, Toshiaki Ichinose.

Funding: None

Conflicts of Interest: The authors declare no conflict of interest.

\section{References}

Birrell, P. J., Zhang, X. S., Pebody, R. G., Gay, N. J., \& De Angelis, D. (2016). Reconstructing a spatially heterogeneous epidemic: Characterising the geographic spread of 2009 A/H1N1pdm infection in England. Scientific Reports, 6, 29004. https://doi.org/10.1038/srep29004

Chen, H. Y., White, D. J., Caraco, T. B., \& Stratton, H. H. (2005). Epidemic and spatial dynamics of Lyme disease in New York State, 1990-2000. Journal of Medical Entomology, 42(5), 899-908. https://doi.org/10.1603/0022-2585(2005)042[0899:EASDOL]2.0.CO;2

Escaff, D., Toral, R., Van den Broeck, C., \& Lindenberg, K. (2018). A continuous-time persistent random walk model for flocking. Chaos, 28, 075507. https://doi.org/10.1063/1.5027734

Fitzgibbon, W. E., Morgan, J. J., Webb, G. F., \& Wu, Y. X. (2019). Spatial models of vector-host epidemics with directed movement of vectors over long distances. Mathematical Biosciences, 312, 77-87. https://doi.org/10.1016/j.mbs.2019.04.003

Gorbalenya, A. E., Baker, S. C., Baric, R. S., De Groot, R. J., Drosten, C., Gulyaeva, A. A., ... Ziebuhr, J. (2000). Severe acute respiratory syndrome-related coronavirus: The species and its viruses - a statement of the Coronavirus Study Group. bioRxiv. 2020. https://doi.org/10.1101/2020.02.07.937862

Haggett, P. (1976). Hybridizing alternative models of an epidemic diffusion process. Economic Geography, 52, 136-146. https://doi.org/10.2307/143360

Kermack, W. O., \& McKendrick, A. G. (1927). A contribution to the mathematical theory of epidemics. Proc. Roy. Soc. of London, 1927, Series A 115, 772, 700-721. https://doi.org/10.1098/rspa.1927.0118

Nicosia, A., Duchesne, T., Rivest, L. P., \& Fortin, D. (2017). A general hidden state random walk model for animal movement. Computational Statistics \& Data Analysis, 105, 76-95. https://doi.org/10.1016/j.csda. 2016.07.009

Reluga, T. C., Medlock, J., \& Galvani, A. P. (2006). A model of spatial epidemic spread when individuals move within overlapping home ranges. Bulletin of Mathematical Biology, 68, 401-416. https://doi.org/10.1007/s11538-005-9027-y

Simoes, J. M. (2005). Spatial epidemic modelling in social networks. AIP Conference Proceedings, 776, 287. https://doi.org/10.1063/1.1985395

Smallman-Raynor, M., Cliff, A., \& Barford, A. (2015). Geographical perspectives on epidemic transmission of Cholera in Haiti, October 2010 through March 2013. Annals of the Association of American Geographers, 105, 665-683. https://doi.org/10.1080/00045608.2015.1050755

Zhou, S. P., Yang, X. L., Wang, X. G., Hu, B., Zhang, L., Zhang, W., ... Shi, Z. L. (2020). A pneumonia outbreak associated with a new coronavirus of probable bat origin. Nature, 579, 270-273. https://doi.org/10.1038/s41586-020-2012-7

\section{Copyrights}

Copyright for this article is retained by the author(s), with first publication rights granted to the journal.

This is an open-access article distributed under the terms and conditions of the Creative Commons Attribution license which permits unrestricted use, distribution, and reproduction in any medium, provided the original work is properly cited. 